\title{
Cooperative effects of soy isoflavones and carotenoids on osteoclast formation
}

\author{
Miki Tadaishi,, Yoriko Nishide, ${ }^{1}$ Yuko Tousen, ${ }^{1}$ Marlena C. Kruger ${ }^{2}$ and Yoshiko Ishimi ${ }^{1, *}$ \\ 'Department of Food Function and Labeling, National Institute of Health and Nutrition, 1-23-1 Toyama, Shinjuku-ku, Tokyo 162-8636, Japan
}

${ }^{2}$ Institute of Food, Nutrition and Human Health, Massey University, Private Bag 11222, Palmerston North 4442, New Zealand

(Received 24 October, 2013; Accepted 6 November, 2013; Published online 25 January, 2014)

\begin{abstract}
Osteoclasts play a major role in bone resorption. Several functional food components, such as soy isoflavones and carotenoids, are reported to inhibit osteoclast formation. However, the cooperative effect of functional foods or their constituents on bone metabolism has not been clarified. This study aimed to investigate the cooperative effect of soy isoflavones and carotenoids on osteoclast formation in vitro using cultures of RAW264 and bone marrow cells in the presence of receptor activator of nuclear factor $\kappa-B$ ligand. In RAW264 cells, treatment with soy isoflavones (genistein or equol) or carotenoids ( $\beta$-carotene) suppressed osteoclast formation. At $10 \mu \mathrm{M}$, genistein and equol inhibited RAW264 cell proliferation but did not affect cell viability. When $10 \mu \mathrm{M}$ genistein or equol was combined with $0.1 \mu \mathrm{M} \beta$ carotene, we observed an additive suppressive effect on osteoclast differentiation. Similar results were observed with bone marrow cell cultures. We found that $10 \mu \mathrm{M}$ of zeaxanthin or lutein suppressed osteoclast formation singly, and further enhanced the suppressive effects of daidzein or genistein when administered in combination. These results suggest that the combination of soy isoflavones and carotenoids have an enhanced suppressive effect on osteoclast formation. This knowledge might be important in planning diet for bone health.
\end{abstract}

Key Words: soy isoflavones, carotenoids, osteoclast formation, bone

$\mathrm{R}$ ecently, the physiological functions of many components in foods have been identified, and the foods containing such components are called 'functional foods'. The concept of functional foods is used to promote health or to reduce the risk of certain diseases, such as osteoporosis. The loss of bone mass associated with aging is a risk factor for bone fracture and will have a profound impact on sustaining a healthy life. Bone is maintained by a balance between bone resorption and bone formation, known as bone remodeling. Osteoporosis is caused by an imbalance in bone turnover whereby the rate of bone resorption exceeds that of bone formation. Osteoclasts, which are responsible for bone resorption, are multinucleated cells derived from hematopoietic stem cells and their differentiation is controlled by various factors, such as hormones, cytokines, and nutrients. ${ }^{(1)}$ Osteoblasts, conversely, are responsible for bone formation and regulate bone resorption by osteoclasts through paracrine secretion of proteins such as osteoprotegerin. ${ }^{(2)}$

Soy isoflavones, such as daidzein and genistein, are present in soybeans and are known to be functional food components. Daidzein, genistein, and the daidzein metabolite, equol, have a similar structure to estrogen and have the ability to bind to estrogen receptors (ERs). ${ }^{(3)}$ A previous study has shown that soy isoflavones may prevent postmenopausal osteoporosis and improve bone strength, ${ }^{(4)}$ suggesting that soy isoflavones and equol have estrogenic actions in vivo. Moreover, there are also reports that soy isoflavones and equol suppress osteoclast formation in vitro. ${ }^{(5,6)}$

Carotenoids are another type of functional food component reported to also prevent reductions in bone density. ${ }^{(7-9)}$ One example is $\beta$-carotene, which is a major precursor to vitamin A. Beta-carotene is metabolized to vitamin A by its cleavage enzyme, $\beta$-carotene oxygenase. Several studies have shown that vitamin A, $\beta$-carotene, and another precursor of vitamin A, $\beta$-cryptoxanthin, all have suppressive effects on osteoclast formation. ${ }^{(10,11)}$ Likewise, non-precursors of vitamin A carotenoids can also suppress osteoclast formation. ${ }^{(10,12,13)}$ For example, lycopene, one of the nonprecursors of vitamin A carotenoids, has antioxidant activity and suppresses osteoclast formation. ${ }^{(13)}$ Zeaxanthin and lutein, two other well-known major non-precursors of vitamin A carotenoids, also have antioxidant activity and have been reported to protect against age-related eye diseases, such as age-related macular degeneration. ${ }^{(14,15)}$ Thus, zeaxanthin and lutein are expected to suppress osteoclast formation similar to lycopene.

The attenuation of bone loss by functional foods or its components has been considered recently. However, the efficacy and safety of a combined intake of functional foods or their constituents has not been adequately addressed. Thus, consideration of the interactions amongst these components is important. The present study investigated the cooperative effect of soy isoflavones and carotenoids on osteoclast formation in vitro.

\section{Materials and Methods}

Reagents. Daidzein and genistein were obtained from Nagara Science (Gifu, Japan). (R)-, $(S)$-Equol was obtained from LC Laboratories (Worburn, MA). Soy isoflavone stock solutions were dissolved in dimethyl sulfoxide (Wako, Osaka, Japan) and used at less than $0.1 \%$ in the medium. Beta-carotene was obtained from Sigma-Aldrich (St. Louis, MO). Zeaxanthin and lutein were obtained from Extrasynthese (Genay, France). Carotenoids were dissolved in tetrahydrofuran (Sigma-Aldrich) to form a stock solution of $10 \mathrm{mM}$. The stock solutions were stored in the dark with nitrogen gas filling at $-80^{\circ} \mathrm{C}$. The concentrations of the carotenoid stock solutions were measured by spectrophotometer and molar extinction coefficient. ${ }^{(14)}$ The carotenoid concentrations were prepared as above and added to the medium at the experimental concentration, according to Lin et al. ${ }^{(16)}$ Each compound was used in the doses and combinations as outlined in the Results section and in the figure legends.

Cell culture for RAW264 cells. Mouse macrophage preosteoclast RAW264 cells were obtained from Riken (Ibaraki, Japan) and were maintained in phenol red-free $\alpha$-MEM containing $10 \%$ fetal bovine serum (FBS) and 1\% penicillin-streptomycin.

*To whom correspondence should be addressed.

E-mail: ishimi@nih.go.jp 

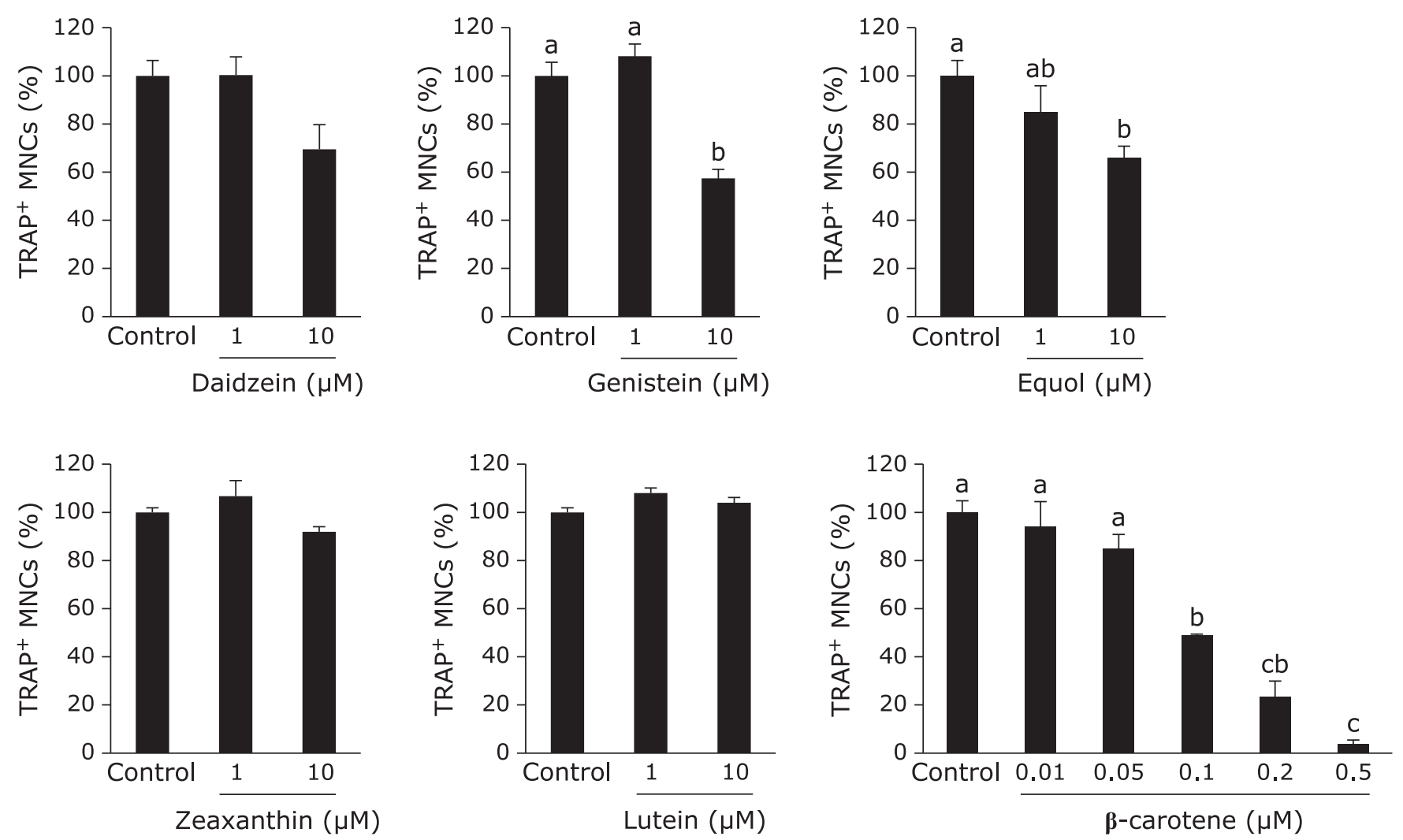

Fig. 1. Effect of single treatment of soy isoflavones and carotenoids on osteoclast formation in RAW264 cell cultures. In the presence of 100 ng/ml RANKL, RAW264 cells were treated with 1 or $10 \mu \mathrm{M}$ of each of the soy isoflavones and carotenoids, with the exception of $\beta$-carotene, which was examined in a dose-responsive manner from 0.01-0.5 $\mu \mathrm{M}$. Figures show the percentage of TRAP-positive multinuclear osteoclast-like cells (MNCs) on day 4 after RANKL induction. Data are expressed as the mean \pm SEM $(n=3)$. The different letters indicate significant differences $(p<0.05)$.

The cell cultures were maintained at $37^{\circ} \mathrm{C}$ in a humidified atmosphere at $5 \% \mathrm{CO}_{2}$ in air. For experiments, cells were seeded and pre-cultured overnight prior to the addition of new media containing the testing reagents. All experimental medium was changed every $48 \mathrm{~h}$.

TRAP-positive multinucleated osteoclast-like cells. For osteoclast formation, $2.5 \times 10^{3}$ cells $/ \mathrm{cm}^{2}$ were seeded into the wells of a 24-well plate. After pre-culture, the medium was changed to media containing $100 \mathrm{ng} / \mathrm{ml}$ recombinant human receptor activator of nuclear factor $\kappa-B$ ligand (rhRANKL; PeproTech, Rocky Hill, NJ) with or without soy isoflavones and carotenoids. The addition of each reagent solvent to the cultured medium was used as a control. After 4 days, the cells were fixed and stained for TRAP activity. ${ }^{(10)}$ Briefly, cells were washed three times with Dulbecco's phosphate-buffered saline (PBS) and fixed in a solution of ethanol-acetone $(1: 1)$. After drying, the cells were stained in $0.1 \mathrm{M}$ sodium acetate buffer ( $\mathrm{pH} 5.0$ ) containing $50 \mathrm{mM}$ sodium tartrate, $0.1 \mathrm{mg} / \mathrm{ml}$ naphthol AS-MX phosphate (SigmaAldrich), and $0.5 \mathrm{mg} / \mathrm{ml}$ fast red violet LB salt (Sigma-Aldrich) at $37^{\circ} \mathrm{C}$. TRAP-positive cells containing three or more nuclei were counted as TRAP-positive multinucleated osteoclast-like cells (MNCs).

Proliferation of RAW 264 cells. Cell proliferation was assessed using 3-(4,5-dimethylthiazol-2-yl)-5-(3-carboxymethoxyphenyl)-2-(4-sulfophenyl)-2H-tetrazolium, inner salt (MTS) and trypan blue staining. To assess for cell proliferation, $2.5 \times 10^{3}$ cells $/ \mathrm{cm}^{2}$ were seeded into the wells of a 96-well plate. To determine cell viability, cells were washed three times with PBS and then assayed using the CellTiter $96^{\circledR}$ AQueous One Solution Cell Proliferation Assay kit (Promega, WI). For trypan blue staining, cells were harvested by trypsinization and stained with
$0.5 \%(\mathrm{w} / \mathrm{v})$ trypan blue in PBS. Trypan blue unstained cells were counted using a hemocytometer under a microscope as viable cells.

Measurement of lactate dehydrogenase activity. Lactate dehydrogenase (LDH) activity in cultured medium was measured as an index of cell death. For LDH activity, $5.0 \times 10^{3}$ cells/well were seeded into each well of a $96-$ well plate and pre-cultured overnight. Cells were then cultured for 2 days in experimental media. Cultured medium was centrifuged for $5 \mathrm{~min}$ at $5,000 \times \mathrm{g}$, and the supernatant was collected for LDH activity measured using the LDH-Cytotoxic Test (Wako).

Total RNA preparation and quantitative real-time reverse transcription- polymerase chain reaction. Cell culture was performed as that described for TRAP-positive MNC culture. On day 3, total RNA preparation and quantitative real-time reverse transcription-polymerase chain reaction (real-time RT-PCR) were performed as described previously. ${ }^{(17)}$ Briefly, total RNA was isolated by Isogen II (Nippon Gene, Tokyo, Japan) by using a primescript RT master mix (Takara, Shiga, Japan). The cDNA was quantified by real-time RT-PCR with SYBR Premix Ex Taq II (Takara). The primer pairs used were as follows: c-Fos forward, 5'-TTACGCCAGAGCGGGAATG-3'; c-Fos reverse, 5'-CTGTCTCCGCTTGGAGTGTATC-3'; nuclear factor of activated T-cells c1 (NFATc1) forward, 5'-GCTTCACCCATTTGCTCCAG-3'; NFATc1 reverse, 5'-ATGGTGTGGAAATACGGTTGGTC-3'; 36B4 forward, 5'-GGCCCTGCACTCTCGCTTTC-3'; 36B4 reverse, 5'-TGCCAGGACGCGCTTGT-3'. Target gene expression levels were normalized to 36B4.

Cell culture for bone marrow cells. Bone marrow cells (BMCs) were obtained from the tibiae of 6- to 8-week-old male ddY mice (Japan SLC, Shizuoka, Japan). Cells were seeded at 

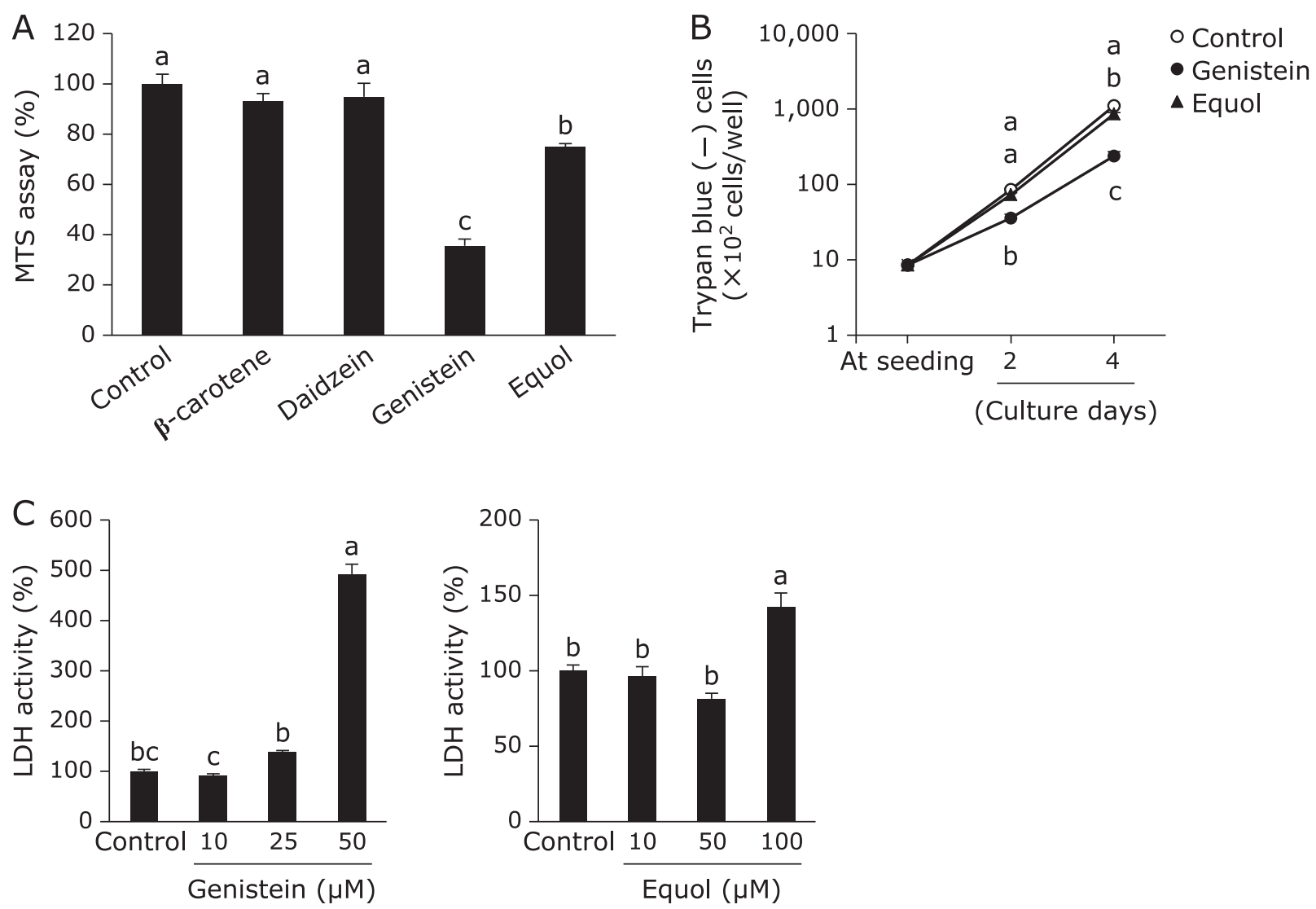

Fig. 2. Effect of single treatment of soy isoflavones and $\beta$-carotene on cell proliferation in RAW264 cell cultures. RAW264 cells were treated $10 \mu \mathrm{M}$ of soy isoflavones and $0.1 \mu \mathrm{M}$ of $\beta$-carotene in the absence of RANKL supplementation. Cell viability was measured by MTS assay (A, at 4 days) and by counting the number of trypan blue unstained (-) cells (B). For an index of cell death, LDH activity in cultured medium was measured with increasing concentrations of genistein or equol for 2 days in the absence of RANKL (C). Data are expressed as the mean \pm SEM ( $n=6)$. The different letters indicate significant differences $(p<0.05)$.

$2.6 \times 10^{5}$ cells $/ \mathrm{cm}^{2}$ in the wells of a 48 -well plate and pre-cultured for $72 \mathrm{~h}$ in phenol red-free $\alpha$-MEM containing $10 \%$ FBS, $1 \%$ penicillin-streptomycin, and $100 \mathrm{ng} / \mathrm{ml}$ recombinant mouse macrophage colony-stimulating factor (M-CSF; R\&D Systems, MN). Osteoclast differentiation was induced by a $100 \mathrm{ng} / \mathrm{ml}$ RANKL addition to the medium. Cells were stained for TRAP at day 6 after induction. The addition of each reagent and TRAP-positive MNC counting were performed in the same manner as described for RAW264 cells above.

Statistical analysis. All experiments were repeated three times, and representative experiments are shown in the figures. Results are the mean \pm standard error of the mean (SEM). Data were analyzed by one-way or two-way analysis of variance (ANOVA). Where differences were significant by one-way ANOVA, groups were compared by the Tukey's test. Statistical significance was defined as $p<0.05$. Statistical analysis was performed using SPSS ver. 19 (IBM, Chicago, IL).

\section{Results}

Effect of soy isoflavones and carotenoids on osteoclast formation in RAW264 cells. In RAW264 cells, $10 \mu \mathrm{M}$ of genistein, $10 \mu \mathrm{M}$ of equol, and $0.1 \mu \mathrm{M}$ of $\beta$-carotene each caused a significant decrease in the percentage of TRAP-positive MNCs in a dose-dependent manner (Fig. 1). Daidzein, zeaxanthin, and lutein did not affect osteoclast formation. The MTS assay showed that genistein and equol caused a decrease in cell proliferation on day 4 (Fig. 2A), and a similar inhibition in cell viability was determined with trypan blue staining at days 2 and 4 (Fig. 2B).
However, compared with days 2 and 4, genistein and equol also increased viable cell number as well as the control. With genistein treatment, the number of trypan blue-negative cells at days 2 and 4 were $3,584 \pm 355$ and $23,805 \pm 2,761$ cells/well, respectively. In equol-treated cultures, the number of trypan blue-negative cells at days 2 and 4 were 7,312 \pm 436 and 86,166 $\pm 3,362$ cells/well, respectively. Additionally, LDH activity in the cultured medium, which is an indicator of cell death, was not increased by $10 \mu \mathrm{M}$ of genistein or equol, but only with higher concentrations of the two compounds (Fig. 2C). These results suggest that genistein and equol inhibit cell proliferation but do not induce cell death at $10 \mu \mathrm{M}$ concentrations.

Cooperative effect of soy isoflavones and carotenoids on osteoclast formation in RAW264 cell cultures. We next tested the combined effect of $10 \mu \mathrm{M}$ of soy isoflavones and $0.1 \mu \mathrm{M}$ of $\beta$-carotene on the number of TRAP-positive MNCs. We found that $\beta$-carotene significantly enhanced the suppressive effect of soy isoflavones on osteoclast formation (Fig. 3A), without affecting cell viability (Fig. 3B). The interactions between the soy isoflavones (daidzein, genistein, and equol) and $\beta$-carotene were not significant for each of these experiments.

Following this, we tested the combined effect of soy isoflavones and $\beta$-carotene on the mRNA expression level of c-Fos and NFATc1 in RAW264 cells with RANKL treatment (Fig. 4). We found that the expression of c-Fos and NFATc1 mRNA was increased by 4.1- and 4.2-fold, respectively, by treatment with RANKL (data not shown). Genistein caused a slight but insignificant decrease in c-Fos mRNA expression whereas daidzein, equol, and $\beta$-carotene caused a significant decrease in 


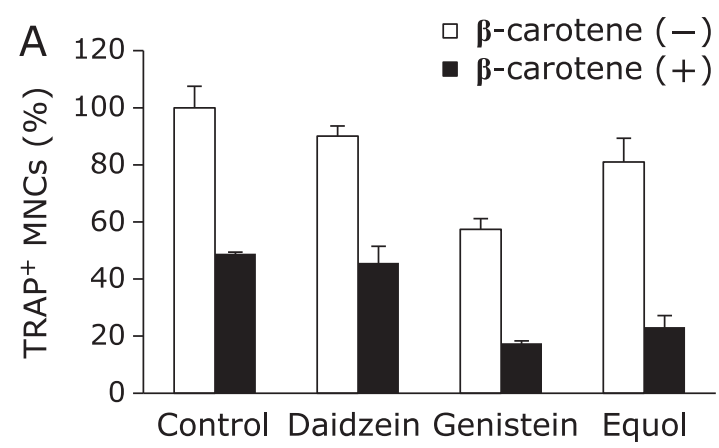

Two-way ANOVA

\begin{tabular}{ccccc}
\hline & \multicolumn{2}{c}{ Factors } & & Interactions \\
\cline { 2 - 3 } & $\begin{array}{c}\text { Soy } \\
\text { isoflavone }\end{array}$ & $\boldsymbol{\beta}$-carotene & & $\begin{array}{c}\text { Soy isoflavone } \\
\times \boldsymbol{\beta} \text {-carotene }\end{array}$ \\
\hline Con. vs Dai. & $p=0.233$ & $p<0.001$ & & $p=0.526$ \\
Con. vs Gen. & $p<0.001$ & $p<0.001$ & & $p=0.244$ \\
Con. vs Eq. & $p<0.01$ & $p<0.001$ & & $p=0.154$ \\
\hline
\end{tabular}

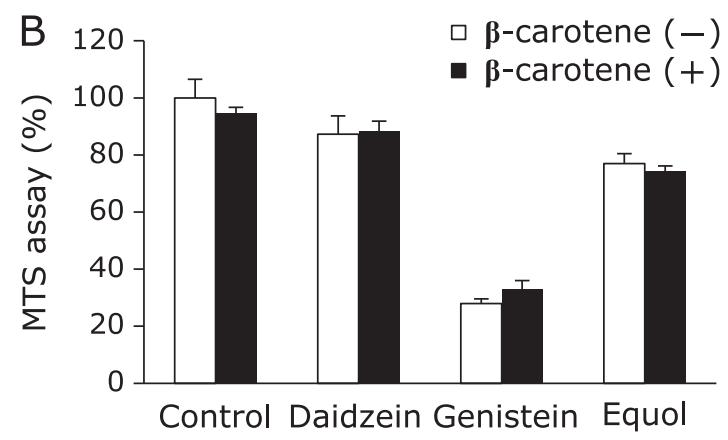

Two-way ANOVA

\begin{tabular}{ccccc}
\hline & \multicolumn{2}{c}{ Factors } & & Interactions \\
\cline { 2 - 3 } & $\begin{array}{c}\text { Soy } \\
\text { isoflavone }\end{array}$ & $\boldsymbol{\beta}$-carotene & & $\begin{array}{c}\text { Soy isoflavone } \\
\times \boldsymbol{\beta} \text {-carotene }\end{array}$ \\
\hline Con. vs Dai. & $p=0.078$ & $p=0.677$ & & $p=0.509$ \\
Con. vs Gen. & $p<0.001$ & $p=0.964$ & & $p=0.184$ \\
Con. vs Eq. & $p<0.001$ & $p=0.313$ & & $p=0.741$ \\
\hline
\end{tabular}

Fig. 3. Cooperative effect of soy isoflavones with $\beta$-carotene on osteoclast formation and cell viability. RAW264 cells were cultured in $100 \mathrm{ng} / \mathrm{ml}$ RANKL containing $10 \mu \mathrm{M}$ of soy isoflavones with (black bars) or without (open bars) $0.1 \mu \mathrm{M}$ of $\beta$-carotene. On day 4 of culture, the number of TRAP-positive multinucleated osteoclast-like cells $(n=3)(\mathrm{A})$ and viable cells $(n=6)$ (B) were determined. Data are expressed as the mean \pm SEM. Statistical analysis was performed using two-way analysis of variance (ANOVA) with the two factors being soy isoflavones (Control: Con., Daidzein: Dai., Genistein: Gen., and Equol: Eq.) and $\beta$-carotene. Significance was determined at $p<0.05$.

its expression (Fig. 4A). By comparison, NFATc1 mRNA expression was significant decreased by all soy isoflavones and $\beta$ carotene (Fig. 4B). The interactions between soy isoflavones (daidzein, genistein, and equol) and $\beta$-carotene were not significant. Therefore, the combination of soy isoflavones and $\beta$ carotene has an additive effect on inhibiting the mRNA expression levels of osteoclast differentiation-related genes.

Cooperative effect of soy isoflavones and carotenoids on osteoclast formation in BMC cultures. We also sought to test these combined effects in a another osteoclast formation system using BMCs pre-differentiated with M-CSF. In differentiated

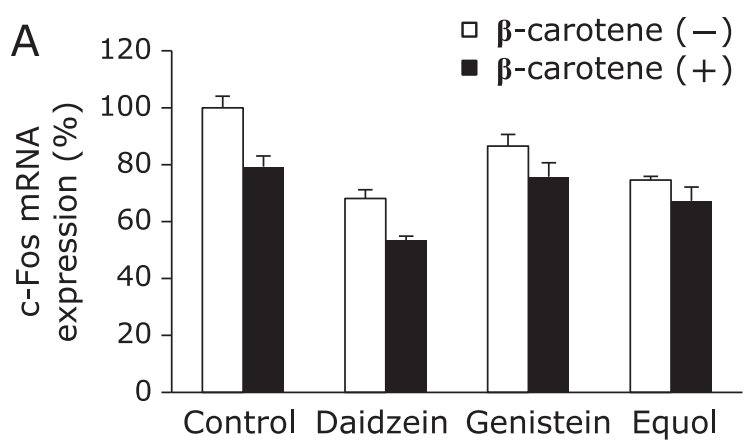

Two-way ANOVA

\begin{tabular}{ccccc}
\hline & \multicolumn{2}{c}{ Factors } & & Interactions \\
\cline { 2 - 3 } & $\begin{array}{c}\text { Soy } \\
\text { isoflavone }\end{array}$ & $\boldsymbol{\beta}$-carotene & & $\begin{array}{c}\text { Soy isoflavone } \\
\times \boldsymbol{\beta} \text {-carotene }\end{array}$ \\
\hline Con. vs Dai. & $p<0.001$ & $p<0.001$ & & $p=0.372$ \\
Con. vs Gen. & $p=0.058$ & $p<0.01$ & & $p=0.257$ \\
Con. vs Eq. & $p<0.001$ & $p<0.01$ & & $p=0.089$ \\
\hline
\end{tabular}

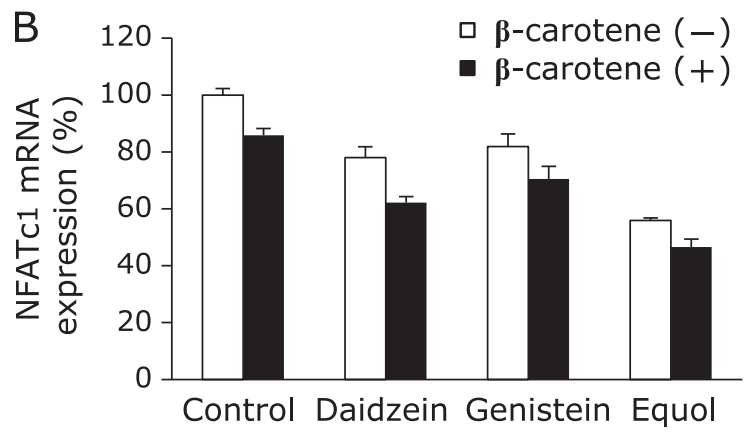

Two-way ANOVA

\begin{tabular}{ccccc}
\hline & \multicolumn{2}{c}{ Factors } & & Interactions \\
\cline { 2 - 3 } & $\begin{array}{c}\text { Soy } \\
\text { isoflavone }\end{array}$ & $\boldsymbol{\beta}$-carotene & & $\begin{array}{c}\text { Soy isoflavone } \\
\times \boldsymbol{\beta} \text {-carotene }\end{array}$ \\
\hline Con. vs Dai. & $p<0.001$ & $p<0.001$ & & $p=0.604$ \\
Con. vs Gen. & $p<0.05$ & $p<0.01$ & & $p=0.475$ \\
Con. vs Eq. & $p<0.01$ & $p<0.01$ & & $p=0.957$ \\
\hline
\end{tabular}

Fig. 4. Cooperative effect of soy isoflavones with $\beta$-carotene on $\mathrm{c}$-Fos and NFATc1 mRNA expression. RAW264 cells were cultured in $100 \mathrm{ng} / \mathrm{ml}$ RANKL containing $10 \mu \mathrm{M}$ of soy isoflavones with (black bars) or without (open bars) $0.1 \mu \mathrm{M}$ of $\beta$-carotene for 3 days. Figures show the percent of c-Fos (A) and NFATc1 (B) mRNA levels relative to control without $\beta$ carotene. Data are expressed as the mean \pm SEM. Statistical analysis was performed using two-way analysis of variance (ANOVA) with the two factors being soy isoflavones (Control: Con., Daidzein: Dai., Genistein: Gen., and Equol: Eq.) and $\beta$-carotene. Significance was determined at $p<0.05$.

BMCs, daidzein, genistein, zeaxanthin, lutein, and $\beta$-carotene all caused a decrease in the number of TRAP-positive MNCs in a dose-dependent manner (Fig. 5). Equol, however, did not significant decrease TRAP-positive MNC number. Following this, we tested the combined effect of $10 \mu \mathrm{M}$ of soy isoflavones with $10 \mu \mathrm{M}$ of zeaxanthin or lutein on the number of TRAP-positive MNCs. We showed that zeaxanthin and lutein both significantly enhanced the suppressive effect of soy isoflavones on osteoclast formation (Fig. 6). The interactions between equol and zeaxanthin were significant. In addition, we tested the cooperative effect of $10 \mu \mathrm{M}$ of soy isoflavones and $0.1 \mu \mathrm{M}$ of $\beta$-carotene on BMC 

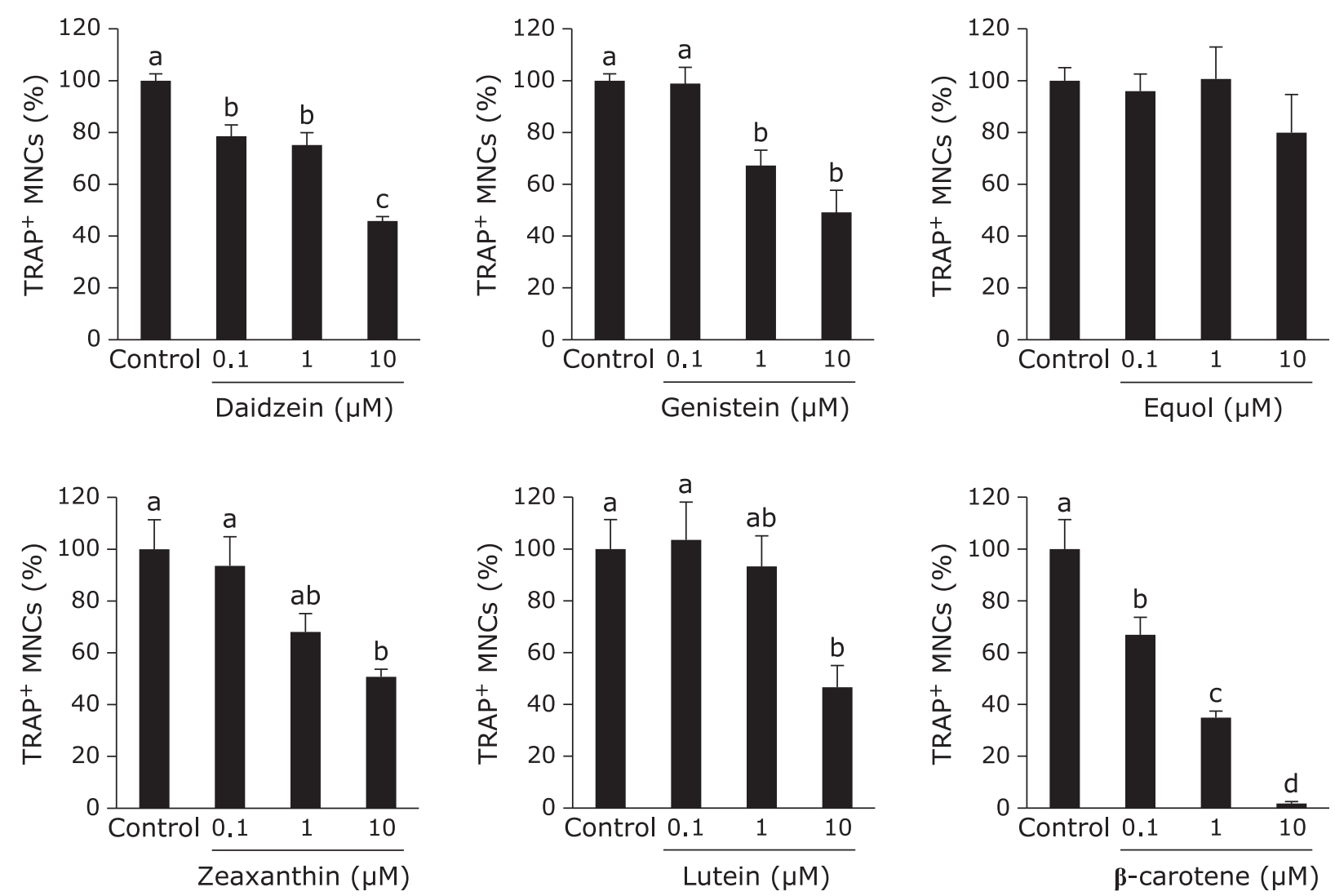

Fig. 5. Effect of single treatment of soy isoflavones and carotenoids on osteoclast formation in bone marrow cell (BMC) cultures. BMCs were precultured for $72 \mathrm{~h}$ in $100 \mathrm{ng} / \mathrm{ml}$ macrophage colony-stimulating factor and then induced to differentiate by the addition of $100 \mathrm{ng} / \mathrm{ml}$ RANKL. Single supplementation with each of the soy isoflavones and carotenoids was administered at the same time. Figures show the percent of TRAP-positive MNCs number on day 6. Data are expressed as the mean \pm SEM $(n=3-5)$. The different letters indicate significant differences $(p<0.05)$.

cultures, and showed that these results mirrored the effects we observed with RAW264 cells (data not shown).

\section{Discussion}

In this study, the cooperative effect of soy isoflavones and carotenoids on osteoclast formation was investigated. Genistein and equol inhibited osteoclast formation and suppressed cell proliferation at $10 \mu \mathrm{M}$ in RAW264 cells. Previous reports have shown that genistein inhibits cell proliferation by causing G2/M cell-cycle arrest and induces apoptosis through an inhibitory effect on protein tyrosine kinase activity. ${ }^{(18,19)}$ However, in our study, LDH activity in the cultured medium (as an indicator of cell death) was unchanged with $10 \mu \mathrm{M}$ of either genistein or equol. This result suggests that, in the present study conditions, genistein and equol inhibit osteoclast formation by suppressing RAW264 cell growth and not by inducing cell death. In addition soy isoflavones suppressed c-Fos and NFATc1 mRNA expression. These genes are key factors in osteoclast formation. ${ }^{(20)}$ Daidzein, genistein, and equol, a metabolite of daidzein, are known as phytoestrogens and are able to bind to ERs. ${ }^{(3)}$ It was previously demonstrated that the soy isoflavones inhibited osteoclast formation by suppressing c-Fos-induced NFATc1 expression and this inhibition could be prevented by estrogen antagonists. ${ }^{(5)}$ These reports suggest that soy isoflavones suppress osteoclastic gene expression through an estrogen-like action.

In RAW264 cells, the addition of $\beta$-carotene, a precursor to vitamin A, also inhibited osteoclast differentiation by suppressing c-Fos and NFATc1 mRNA expression, without affecting cell proliferation. Previous reports have shown that retinoic acid, an active form of vitamin A, has a suppressive effect on osteoclast formation and NFATc1 expression via nuclear retinoic (RARs). ${ }^{(10,21)}$ Therefore, there is possibility that $\beta$-carotene acts on osteoclast formation through activation of the NFATc1 pathway mediated by RARs. The combination of soy isoflavones with $\beta$ carotene enhanced the suppressive effect on osteoclast formation and mRNA expression level of genes associated with osteoclast differentiation. There were no significant interactions between soy isoflavones and $\beta$-carotene as determined in the two-way ANOVA. Thus, soy isoflavones and $\beta$-carotene seemed to have an additive effect on inhibition of osteoclast formation in RAW264 cells.

Zeaxanthin and lutein were shown to suppress osteoclast formation in BMCs. Soy isoflavones in conjunction with zeaxanthin or lutein had an additive effect on osteoclast formation, although the mechanism could not be fully clarified in the present research. Carotenoids, such as zeaxanthin and lutein, have high antioxidant activity and previous studies have shown that antioxidants suppress osteoclast formation. ${ }^{(14,22,23)}$ Thus, zeaxanthin and lutein may have suppressed osteoclast formation through their antioxidant activity.

Zeaxanthin and lutein, however, did not produce the same effect in RAW264 cells as in BMCs. There are numerous differences between osteoclasts derived from BMC and RAW264 cell cultures. BMC cultures include various cell types, including hematopoietic stem cells and marrow stromal cells. The marrow stromal cells have the ability to differentiate into osteoblasts, adipocytes, myocytes, and chondrocytes. Thus, there are not only osteoclast 


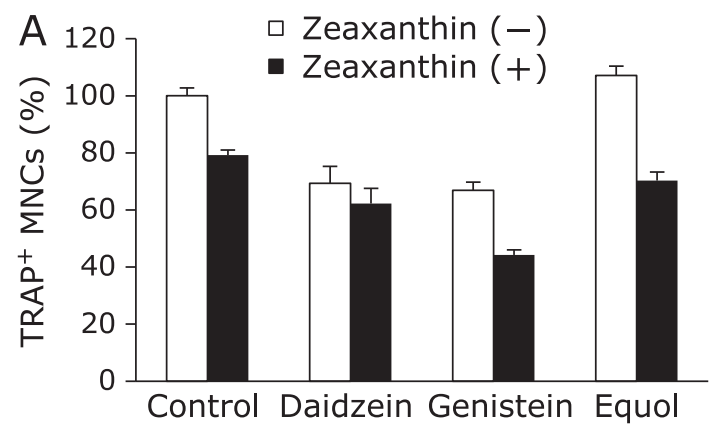

Two-way ANOVA

\begin{tabular}{ccccc}
\hline & \multicolumn{2}{c}{ Factors } & & Interactions \\
\cline { 2 - 3 } & $\begin{array}{c}\text { Soy } \\
\text { isoflavone }\end{array}$ & Zeaxanthin & & $\begin{array}{c}\text { Soy isoflavone } \\
\times \text { Zeaxanthin }\end{array}$ \\
\hline Con. vs Dai. & $p<0.001$ & $p<0.01$ & & $p=0.128$ \\
Con. vs Gen. & $p<0.001$ & $p<0.001$ & & $p=0.697$ \\
Con. vs Eq. & $p=0.738$ & $p<0.001$ & & $p<0.01$ \\
\hline
\end{tabular}

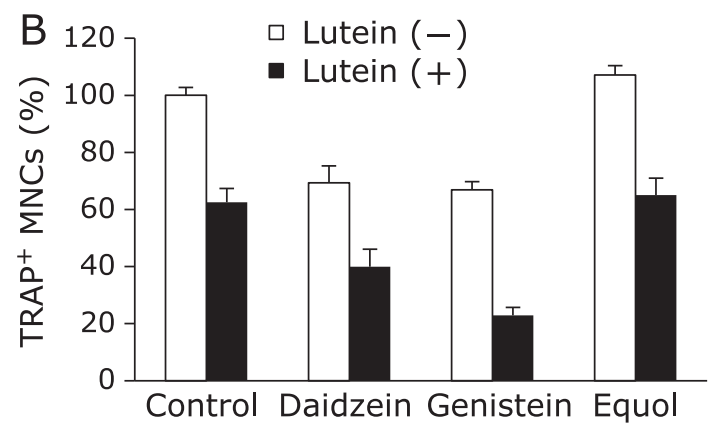

Two-way ANOVA

\begin{tabular}{ccccc}
\hline & \multicolumn{2}{c}{ Factors } & & Interactions \\
\cline { 2 - 3 } & $\begin{array}{c}\text { Soy } \\
\text { isoflavone }\end{array}$ & Lutein & & $\begin{array}{c}\text { Soy isoflavone } \\
\times \text { Lutein }\end{array}$ \\
\hline Con. vs Dai. & $p<0.001$ & $p<0.001$ & & $p=0.437$ \\
Con. vs Gen. & $p<0.001$ & $p<0.001$ & & $p=0.353$ \\
Con. vs Eq. & $p=0.288$ & $p<0.001$ & & $p=0.605$ \\
\hline
\end{tabular}

Fig. 6. Cooperative effect of soy isoflavones with zeaxanthin or lutein on osteoclast formation in bone marrow cell (BMC) cultures. BMCs were pre-cultured for $72 \mathrm{~h}$ in $100 \mathrm{ng} / \mathrm{ml}$ macrophage colony-stimulating factor and then induced to differentiate with $100 \mathrm{ng} / \mathrm{ml}$ RANKL. Supplementation with $10 \mu \mathrm{M}$ of soy isoflavones with or without zeaxanthin (A) or lutein (B) was administered at the same time as RANKL addition. Data are expressed as the mean $\pm \operatorname{SEM}(n=5)$. Statistical analysis was performed using two-way analysis of variance (ANOVA) with the two factors being soy isoflavones (Control: Con., Daidzein: Dai., Genistein: Gen., and Equol: Eq.) and carotenoids (zeaxanthin or lutein). Significance was determined at $p<0.05$.

precursors, but also other types of cellular precursors in the BMC culture system. Because osteoclast formation is regulated by cellcell interactions with osteoblasts in particular, ${ }^{(2,24,25)}$ it is possible that zeaxanthin and lutein had an effect on osteoclast formation via these other cells in the culture.

In conclusion, this study demonstrates that the combination of soy isoflavones and carotenoids has a compound suppressive effect on osteoclast formation. This is the first report to demonstrate the possible cooperative effects of soy isoflavones and carotenoids on osteoclast formation. These findings are of importance as they indicate a possible additive effect of the functional foods containing these components on bone metabolism and the prevention of bone loss.

\section{Acknowledgments}

This work was supported by the Strategic Japanese (Japanese Science and Technology Agency) - New Zealand (Ministry of Business, Innovation and Employment) Cooperative Program on "Functional Food".

\section{Conflict of Interest}

No potential conflicts of interest were disclosed.

\section{References}

1 Udagawa N, Takahashi N, Akatsu T, et al. Origin of osteoclasts: mature monocytes and macrophages are capable of differentiating into osteoclasts under a suitable microenvironment prepared by bone marrow-derived stromal cells. Proc Natl Acad Sci US A 1990; 87: 7260-7264.

2 Simonet WS, Lacey DL, Dunstan CR, et al. Osteoprotegerin: a novel secreted protein involved in the regulation of bone density. Cell 1997; 89: 309-319.

3 Kostelac D, Rechkemmer G, Briviba K. Phytoestrogens modulate binding response of estrogen receptors alpha and beta to the estrogen response element. J Agric Food Chem 2003; 51: 7632-7635.

4 Taku K, Melby MK, Nishi N, Omori T, Kurzer MS. Soy isoflavones for osteoporosis: an evidence-based approach. Maturitas 2011; 70: 333-338.

5 Karieb S, Fox SW. Phytoestrogens directly inhibit TNF- $\alpha$-induced bone resorption in RAW264.7 cells by suppressing c-fos-induced NFATc1 expression. J Cell Biochem 2011; 112: 476-487.

6 Ohtomo T, Uehara M, Peñalvo JL, et al. Comparative activities of daidzein metabolites, equol and O-desmethylangolensin, on bone mineral density and lipid metabolism in ovariectomized mice and in osteoclast cell cultures. Eur J Nutr 2008; 47: 273-279.

7 Sahni S, Hannan MT, Blumberg J, Cupples LA, Kiel DP, Tucker KL. Protective effect of total carotenoid and lycopene intake on the risk of hip fracture: a 17-year follow-up from the Framingham Osteoporosis Study. $J$ Bone Miner Res 2009; 24: 1086-1094.

8 Sugiura M, Nakamura M, Ogawa K, Ikoma Y, Yano M. High serum carotenoids associated with lower risk for bone loss and osteoporosis in postmenopausal Japanese female subjects: prospective cohort study. PLoS One 2012; 7: e52643.

9 Wattanapenpaiboon N, Lukito W, Wahlqvist ML, Strauss BJ. Dietary carotenoid intake as a predictor of bone mineral density. Asia Pac J Clin Nutr 2003; 12: 467-473.

10 Ishimi Y, Ohmura M, Wang X, Yamaguchi M, Ikegami S. Inhibition by carotenoids and retinoic acid of osteoclast-like cell formation induced by bone-resorbing agents in vitro. J Clin Biochem Nutr 1999; 27: 113-122.

11 Yamaguchi M, Uchiyama S. beta-Cryptoxanthin stimulates bone formation and inhibits bone resorption in tissue culture in vitro. Mol Cell Biochem 2004; 258: $137-144$.

12 Das SK, Ren R, Hashimoto T, Kanazawa K. Fucoxanthin induces apoptosis in osteoclast-like cells differentiated from RAW264.7 cells. J Agric Food Chem 2010; 58: 6090-6095.

13 Rao LG, Krishnadev N, Banasikowska K, Rao AV. Lycopene I-effect on osteoclasts: lycopene inhibits basal and parathyroid hormone-stimulated osteoclast formation and mineral resorption mediated by reactive oxygen species in rat bone marrow cultures. J Med Food 2003; 6: 69-78.

14 Miller NJ, Sampson J, Candeias LP, Bramley PM, Rice-Evans CA. Antioxidant activities of carotenes and xanthophylls. FEBS Lett 1996; 384: 240 242.

15 Abdel-Aal El-SM, Akhtar H, Zaheer K, Ali R. Dietary sources of lutein and zeaxanthin carotenoids and their role in eye health. Nutrients 2013; 5: 11691185.

16 Lin CY, Huang CS, Hu ML. The use of fetal bovine serum as delivery vehicle to improve the uptake and stability of lycopene in cell culture studies. Br J Nutr 2007; 98: 226-232.

17 Nishide Y, Tousen Y, Inada M, Miyaura C, Ishimi Y. Bi-phasic effect of equol on adipocyte differentiation of MC3T3-L1 cells. Biosci Biotechnol 
Biochem 2013; 77: 201-204.

18 Matsukawa Y, Marui N, Sakai T, et al. Genistein arrests cell cycle progression at G2-M. Cancer Res 1993; 53: 1328-1331.

19 Spinozzi F, Pagliacci MC, Migliorati G, et al. The natural tyrosine kinase inhibitor genistein produces cell cycle arrest and apoptosis in Jurkat Tleukemia cells. Leuk Res 1994; 18: 431-439.

20 Takayanagi H, Kim S, Koga T, et al. Induction and activation of the transcription factor NFATc1 (NFAT2) integrate RANKL signaling in terminal differentiation of osteoclasts. Dev Cell 2002; 3: 889-901.

21 Balkan W, Rodríguez-Gonzalez M, Pang M, Fernandez I, Troen BR. Retinoic acid inhibits NFATc1 expression and osteoclast differentiation. $J$ Bone Miner Metab 2011; 29: 652-661.

22 Moon HJ, Ko WK, Han SW, et al. Antioxidants, like coenzyme Q10, selenite, and curcumin, inhibited osteoclast differentiation by suppressing reactive oxygen species generation. Biochem Biophys Res Commun 2012; 418: $247-253$.

23 Lee JH, Kim HN, Yang D, et al. Trolox prevents osteoclastogenesis by suppressing RANKL expression and signaling. $J$ Biol Chem 2009; 284: $13725-13734$.

24 Pearse RN, Sordillo EM, Yaccoby S, et al. Multiple myeloma disrupts the TRANCE/ osteoprotegerin cytokine axis to trigger bone destruction and promote tumor progression. Proc Natl Acad Sci U S A 2001; 98: 11581-11586.

25 Roux S, Meignin V, Quillard J, et al. RANK (receptor activator of nuclear factor-kappaB) and RANKL expression in multiple myeloma. Br J Haematol 2002; 117: 86-92. 